\title{
Voice-based protocols for Mobile Ad Hoc Networks: Challenges, Design Principles, and Implementations
}

\begin{abstract}
$\underline{\text { Ala' F. A. Aburumman }}$
School of Information Technology and Mathematical Sciences, University of South Australia, South Australia Email: jordan.aburumman@unisa.edu.au

Abstract: With the increasingly popularity of mobile devices (e.g. iPhones and iPads), Mobile Ad hoc Networks (MANETs) has emerged as one of the topical research areas in recent years. The special characteristics of MANETs (i.e. infrastructure-less and self-configuring) provide a flexible way of connecting mobile devices. Such implementation can provide the means of communications where telecommunication infrastructure is inattentive (Out-of-range, out of credit). Due to the inherent characteristics of MANETs (e.g. self-configuration of IP addresses), implementing Voice over IP (VoIP) services over MANETs remains an ongoing research challenge. In this paper, we highpoint the challenges of implementing VoIP, recommend design principles for such implementation and discuss some implementations. This research is based on an expert advice with over 15 years' experience in the area of computer network and around 10 years on intensive research in the area of adapting voice-based protocol for MANETs.
\end{abstract}

Keywords: $\quad$ Mobile Ad hoc Networks (MANETs), Voice over IP (VoIP), voice-based protocol 


\section{INTRODUCTION}

Wireless Ad hoc Networks are collections of autonomous nodes forming a temporary network without the aid of any centralized administration. One of the challenges facing MANETs is Quality of Service (QoS) control for multimedia applications (Corson \& Macker, 1999).

The telephony world has evolved from an analog telephone network to a digital circuitswitching network over the last couple of decades. Recently, we are riding a new wave to move from circuit-switching to packetswitching technology for carrying both voice

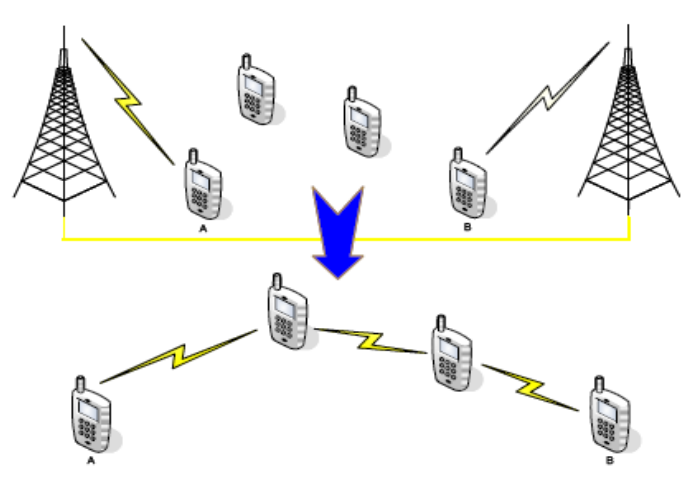

Figure 1. Moving from infrastructure-based networks to Mobile Ad hoc Networks. and data over IP networks. This move led to the emergence of VoIP technology, where reduced long distance tariffs, eliminated access charges and addedvalue services played a major role in promoting the technology (Keromytis, 2011).

Due to the increasing popularity of $802.11 / \mathrm{Wi}$-Fi enabled devices with more powerful capabilities such as smart mobile devices, Ad hoc networks are used to support various applications that run on such devices. One such application is inexpensive communication using VoIP application on Wi-Fi-enabled devices. However, the pre-infrastructure networks are always a way to charge you and control your communication with others people. The implementation of voice protocol over MANETs would also be a good option to communicate with others in case you don't have network coverage in any area.

Therefore, it is necessary that VoIP signaling services are modified in order to be used in decentralized infrastructure-less networks. To do so, the existing voice over interested protocols could be modified to be utilized in a peer-to-peer (P2P) communication environment, and without compromising on availability, flexible design and accepted call ratio (El Sawda \& Urien, 2006). This will lead to the ability to communicate free of cost as no infrastructure is involved. It also means you are never "Out of Range" as long as people exist around you.

While most work done in VoIP technology concentrated on wired, infrastructure-based networks (Internet, enterprise networks) the question arises whether VoIP technology could also be used in wireless, noninfrastructure environments, such as Mobile Ad hoc Networks (MANETs). In the case of a university campus, one could imagine phone calls to be routed through ad hoc networks whenever available, which is free of costs as depicted in Figure 1.

In this paper, we highpoint the challenges of implementing VoIP, recommend some design principles for such implementation and discuss some implementations

\section{MOTIVATION, CHALLENGES AND DESIGN PRINCIPLES}

\subsection{Motivation}

Wireless devices play an important role in our daily communications. Applications are deployed in the wireless environment, such as Voice over Internet Protocol (VoIP). VoIP is a general term for the family set of technologies and protocols used to deliver multimedia (voice and video) over Internet Protocol (IP) networks, instead of the Public Switched Telephone Network (PSTN). In IP-based networks, widely known signaling protocols include H.323 - defined by the International Telecommunication Union (ITU) - and the Session Initiation Protocol (SIP) - defined by the Internet Engineering Task Force (IETF). SIP is increasingly popular (and perhaps overtaking H.323 as the protocol-of-choice) due to SIP's flexibility and relative simplicity (Keromytis 2011). Due to the increasing popularity of $802.11 / \mathrm{Wi}$-Fi enabled devices with more computational powers such as smart mobile devices (e.g. iOS and Android mobile devices), ad hoc networks are used to support many different applications such as (mobile) VoIP applications. Due to the popularity and wide range of (mobile) VoIP applications and 802.11/Wi-Fi enabled devices, deploying (mobile) VoIP applications over ad hoc networks would be timely. For example, students in a campus building need to communicate on-the-fly 
and cheaply. With the availability of Wi-Fi devices, VoIP applications can communicate directly instead of using legacy means of established communication. (Kumar 2006)

To provide pervasive VoIP communication, existing VoIP signaling services and services need to be modified to be used in decentralized infrastructure-less networks, and provide availability and scalability.

The implantation of voice protocol over MANETs would provide a voice service in case of emergency (out of range or service). This implementation can be used for different purposes such as military and emergency management situations where no infrastructure networks are available to communicate through. (Sparks 2006)

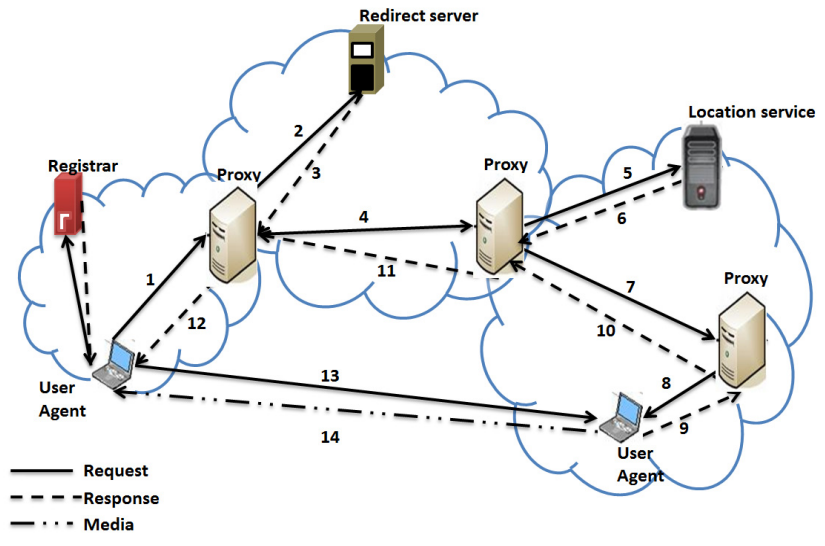

Figure 2. VoIP Overly Network architecture

In a military context, the voice service distribution over MANET would provide an isolated secure communication to communicate within the battlefield where the infrastructure networks cannot be trusted to be used or may be unavailable. Most-likely the distribution of voice services for military use would be much easier to be implemented, due to the fact that the security mechanisms and secret codes can be pre-agreed prior to the distribution of the platoons in the battlefield which makes it easier to be managed and secured.

For commercial purposes, the distribution of voice service over MANET is much harder to implement considering that the involved parties are anonymous and the network is accessible by all. Therefore, this implementation should consider a good adaptation of voice service over MANET in a secured way. This adaptation should also consider a service discovery component to announce the service. The security part of implementing voice service over MANET should be solid and light to be able to fulfil the security requirements as well as keeping the network performance as high as possible.

\subsection{Challenges}

The main challenge with adapting VoIP for MANETS is its reliance on a centralized infrastructure as shown in Figure 2. Tactlessly, MANETs are inherently decentralized, mobile and error-prone in transmission. This means that to use SIP in MANETs, a replacement for all centralized entities in the SIP architecture has to be found.

The scalability is also a challenging issue, as the increase number of nodes joining the network would definitely impact the overall performance of the network which could potentially cause in bringing the network to a halt. So a node management algorithm could be challenging, especially when dealing with an autonomous, selforganizing network such as MANETs.

Another important challenge is security. In such autonomous environment, a compromised node in the network could potentially cause a dramatic impact on the network privacy and performance. The lack of a centralized point makes the issue even more challenging. The integration of a system to adapt the conflicted nature of the protocol and the network itself in a secured manner is needed.

Furthermore, routing protocols is also considered a challenge of high significance. The choice of an appropriate routing protocol to suit such adaptation could be quite challenging.

\subsection{Design Principles}

Implementing and/or adapting a voice- based protocol must first consider the challenges. This will feed into the design principles on for such autonomous environment, and based on over 15 years of experience in this field we could advise following principles:

- Conduct a critical analysis of existing VoIP services, particularly those relating to the potential deployment over MANETs.

- Develop/adapt enhanced compatible routing algorithms for such implementation.

- Develop and validate an adaptive, scalable and secure SIP solution for MANETs. 
- Consider the scalability factor for implementing voice-based service over MANET, within the constraints of the existing network.

- Design an algorithms for Ad hoc node peers to establish calls over the decentralized environment of Ad hoc networks based on SIP; Overcoming existing limitations of relying on static, fixed, and centralized entities; Preventing unnecessary expensive overheads (e.g. eliminating the need to distribute all SIP functionalities over the entire network) without affecting scalability or resulting in higher energy and bandwidth consumption; and Providing a compatible solution complying with the standard SIP.

This could be done using various simulation environment such as Ns2, Ns3, GlomoSIM and using tools to help simulate algorithms such as MATLAB ${ }^{\circledR}$ or formal methods.

\section{IMPLEMENTATIONS}

This section briefly highlights the most relevant and recent implementations found in literature that relates to implement a voice service over MANETs.

In an early implementation, Leggio et al. proposed a fully distributed location service to locating SIP users in as small scaled network to avoid a single point of failure. This is done using by embedding a sundet of SIP proxy and registrar server functionality in all nodes. (Leggio et al. 2005)

Another implementation in the routing protocol aspect of such implementation was produced in 2013, presents two routing protocols:(1) Least delay, Interference Aware Multipath Routing protocol (LIMR) and (2) Shortest path, Interference Aware Multipath Routing protocol (SIMR). Both proposed protocols are designed to reduce the influence of interference between the selected node-disjoint multipath schemes, by selecting node-disjoint routes with the minimal interference between them. In both protocols, we use a new technique in order to reduce the control packets overhead, while enabling the destination node of collecting the required information. This work was simulated using GlomoSIM. (Alwadyah et al. 2013)

Bai and team 2010, uses a test-bed infrastructure to form a distributed wireless multimedia network based on SIP protocol that allow text, voice and video communication to both wired and wireless devices. Utilizing Authentication, Authorization, and Accounting (AAA) server and SIP server, Bai approach still require a centralized controlled authentication which is not application in an Ad hoc network and other decentralized environment. (Bai et al. 2010)

Focusing on the two different MANET environments, which are standalone MANETs and Multihop Cellular Network (MCNs), Bah's research addressed the service provisioning aspects in both environment. Bah's proposed a business model that defines the relationship and interfaces of MANET and the service provision in the MANET. The approach is tailored for closed environment setting with the voice service and security mechanisms that are agreed in advance. (Bah et al. 2010)

In 2011, Kagoshima et al. MANET proposed an emulator architecture and local multipath routing suitable for SIP services. MANET emulator implementation confirmed the correct operation of a SIP service from the rise of a request for session establishment, the establishment of voice packets, to the end of the session. They also tested that the local multipath routing provides a high probability of retaining the required path using an enhanced adaptive AODV routing protocol adaptive considering SIP service. (Kagoshima, Kasamatsu, \& Takami, 2011)

More recent in 2012, Alshingiti proposed an enhanced security mechanism for SIP over ad hoc networks. In her research, an extension to the SIP header was introduced to enhance its security for ad hoc networks. This is done by combining Cryptographically Generated Addresses (CGA) with the social network paradigm to provide authentication and message integrity. (Alshingiti et al. 2012)

Todoroki et al. proposed and implemented architecture for MANET emulator for SIP services deployment called SIP_MANET emulator. This architecture supports real-time communication, node mobility, and P2P communication. (Todoroki et al. 2012)

In 2012, we presented a secure nomination-based solution to implement SIP functionality in Ad hoc networks by combining Distributed SIP Location Service with two security techniques, namely; the Digest 
Authentication Access (DAA) and Simple/ Multipurpose Internet Mail Extensions(S/MIME). Both DAA and S/MIME are used to provide secure log in service for users and data exchanged between proxies, respectively. In the proposed solution, a node is elected to be a proxy server (PS) that handles SIP functionality and another node, Change D'affair (CD), is elected to be a backup for the server. The proxy is set to be the first node in the network, and then it will broadcast an election message to select a $\mathrm{CD}$ to be the next proxy after the PS delivers the task to the elected CD. (Aburumman et al. 2012)

In 2013, we demonstrated how we can adapt the widely used Session Initiation Protocol (SIP) (a signaling protocol used to establish, manage and tear a VoIP session) over MANETs using the Nomination-based mechanism. Our proposed solution employs two security mechanisms to form the underlying model of adapting SIP service over MANETs. We then simulated the setup under different conditions and evaluated the results using various metrics. The proposed algorithms we demonstrated using formal methods and MATLAB ${ }^{\mathbb{2}}$. (Aburumman et al. 2013)

In 2014, Almobaideen et al. proposed an adapted and semi distributed SIP protocol that works using clustered MANETs (referred to as FCSIP). In FCSIP, a new role for SIP server was introduced, where the SIP server also acts as a cluster-head to be the discovery servers to allow SIP agents to get information about other clients in the SIP cluster. It was claimed that such implementation would perform better than the fully-distributed SIP protocol over MANETs. (Almobaideen et al. 2014)

In 2015 , we presented a solution addressing the scalability limitation in a domain-based distribution of SIP services. We used a dynamic clustering to maximize the usage of resources to facilitate the deployment of SIP over MANETs. Our simulation results demonstrated that scalability of SIP service is increased, while minimizing the overheads by eliminating or dividing the workload among servers (i.e., cluster heads). However, security was not considered in this work. (Aburumman et al. 2015)

In 2016, we proposed a cross-domain Session Initiation Protocol (SIP), a widely used voice over Internet Protocol (VoIP) protocol, solution for MANETs using dynamic clustering by extending the scheme of Aburumman and Choo. Our enhanced solution allows us to scale across domains, and deal with outbound requests, using the reputation method. Advantages of this solution include avoiding the shortcomings associated with centralized approaches, such as a single point of failure. To demonstrate the utility of the solution, we simulate and evaluate the proposed solution under different conditions and using Ns3. (Aburumman et al. 2016)

It is clear from the literature that improving the scalability and security of SIP services on MANETs is an ongoing research challenge. This is not surprising as voice- based protocol's relies on the resources of server functions, and unfortunately in a MANET environment, servers play a limiting role. As the size of the network increases, the load on the servers increases; consequently, this affects the level of reliability and availability. The dynamic, unpredictable and self-configuring nature of MANETs also complicates efforts to maximize the scalability and security of SIP services over MANETs.

\section{CONCLUSIONS AND RECOMMENDATIONS}

This paper presents and highpoints the challenges of implementing VoIP for MANETs, recommends design principles for such implementation and discuss some implementations. Successful implementation of such adaptation could be a life changing tool in circumstance where no telecommunication infrastructure exists. Imagine the ability to use an application to freely make calls with no bounds to any infrastructure to charge you for using their components. Better yet, this could potentially provide us with the means to communicate in case of emergencies (out of range, out of credits) when it really matters!

As noticed in literature the implementation of voice service over MANETs did not cover all the implementation factors to make a success model of such implementation, lacking either scalability or security factors; these factors are very important for implementing voice-based service over MANETs, if service meant to survive, This makes this research topic an open challenge for researcher who are interested in making a contribution to the humanity. So, this paper can be used as a roadmap for implementation of inspiration

Future work includes implementing a prototype to be used on real life cases and experiments. 
Aburumman, Voice-based protocols for Mobile Ad Hoc Networks: Challenges, Design Principles, and Implementations

\section{REFERENCES}

Alshingiti, M. (2012). Security Enhancement for SIP in Ad Hoc Networks.Ph.D. Thesis, Carleton University.

Aburumman A., Choo K-K R., and Lee I. (2013). Nomination-based session initiation protocol service for mobile ad hocnetworks. In Proceedings of 22nd National Conference of the Australian Society for Operations Research (ASOR2013) , pp. 149-155, The Australian Society for Operations Research (2013).

Abdullah L., Almomani I., and Aburumman A. (2013). Secure cluster-based SIP service over Ad hoc networks. In Proceedings of IEEE Jordan Conference on Applied Electrical Engineering and Computing Technologies (AEECT2013), pp. 1-7, IEEE (2013).

Aburumman A., and Choo K-K R. (2015). A Domain-Based Multi-cluster SIP solution for Mobile Ad hoc network. In Proceedings of International ICST Conference on Security and Privacy in Communication Networks (SecureComm2014), Springer (2015).

Aburumman A., Seo W. J., Islam R., Khan M. K. and Choo K-K R. (2016). A Secure Cross-Domain SIP Solution for Mobile Ad Hoc Network using Dynamic Clustering. In Proceedings of International ICST Conference on Security and Privacy in Communication Networks (SecureComm 2015), Springer (2016).

Almobaideen, W., Kubba, N., \& Awajan, A. W. (2014). FCSIP: Fuzzy and Cluster Based SIP Protocol for MANET. In Proceedings of International Conference on Next Generation Mobile Apps, Services and Technologies (NGMAST 2014), pp. 169-174, IEEE (2014).

Alwadiyeh ES, Aburumman A and Choo K-K R (2013). Interference-aware multipath routing protocols for mobile ad hoc networks. In Gaertner, P., Bowden, F., Piantadosi, J. and Mobbs, K. (eds) 22nd National Conference of the Australian Society for Operations Research (ASOR 2013). The Australian Society for Operations Research, Adelaide, pp. 156-162, 1-6 December 2013.

Bai, Y., Aminullah, S., Han, Q., Wang, D., Zhang, T., \& Qian, D. (2007). A novel distributed wireless VoIP server based on SIP. In Proceedings of International Conference on Multimedia and Ubiquitous Engineering (MUE 2007), pp. 958-962, IEEE (2007).

Bah, S. (2010). SIP servlets-based service provisioning in MANETs, Concordia University.

Corson, S., and J., Macker (1999). Mobile Ad hoc Networking (MANET):Routing Protocol Performance Issues and Evaluation Considerations. IETF RFC: 2501.

EI Sawda, S., and P. Urien (2006). SIP Security Attacks and Solutions: A state-of-the-art review. Paper presented at the Information and Communication Technologies, 2006, ICTTA '06. 2nd, Damascus, April 24-28.

Kagoshima, T., D.Kasamatsu, and T. Kazumasa (2011). Architecture and emulator in ad hoc network for providing P2P type SIP_VoIP services. Paper presented at the TENCON 2011 - 2011 IEEE Region 10 Conference, Bali, November 21-24.

Keromytis, A.D. (2011). A Comprehensive Survey of Voice over IP Security Research. IEEE Communications Surveys \& Tutorials, 14(2), 514-537.

Kumar, A. (2006). An overview of voice over internet protocol (voip). Rivier College Online Academic Journal, 2(1), 1-13.

Sparks, R. (2007). SIP: basics and beyond. Queue, 5(2), 22-33.

Todoroki, H., T. Kagoshima, D. Kasamatsu, and K. Takami (2012). Implementation of a peer-to-peer-type SIP client application on a MANET emulator. Paper presented at the TENCON 2012 - 2012 IEEE Region 10 Conference, Cebu, November 19-22. 